\title{
A transformação inteligente das cidades brasileiras na perspectiva da governança de TIC
}

\section{Luiz Claudio Diogo Reis ${ }^{1}$, Flavia Cristina Bernardini ${ }^{2}$, Claudia Cappelli ${ }^{3}$, Simone Bacellar Leal Ferreira ${ }^{1}$}

\author{
${ }^{1}$ Instituto de Informática - Universidade Federal do Estado do Rio de Janeiro \\ (UNIRIO) Av. Pasteur, 458 - Urca CEP 22290240 - Rio de Janeiro - RJ - Brasil \\ ${ }^{2}$ Departamento de Computação - Universidade Federal Fluminense (UFF) \\ - Niterói - Rio de Janeiro - RJ - Brasil \\ ${ }^{3}$ Instituto Instituto de Matemática e Estatística - Universidade do Estado do Rio \\ de Janeiro (UERJ) \\ \{luiz.reis, simone\}@uniriotec.br, flavia.bernardini@ic.uff.br, \\ claudia.cappelli@gmail.com
}

\begin{abstract}
In the context of digital transformation, cities are adopting smart city initiatives, in which ICT represents a transversal enabler. Several studies characterized cities' smartness, but few focused on ICT governance. Thus, this research analyzed ICT governance practices in fifteen Brazilian cities best ranked in the Smart City Connected index. The methodology encompassed cities' official websites, transparency portals, and requests based on Brazilian Access Information Law. The results evidenced that most of the cities lack a smart city program approach and ICT governance practices implementation. Additionally, some cities were not compliant with the Information Access Law.
\end{abstract}

Resumo. No contexto da transformação digital, os municípios estão adotando iniciativas de cidades inteligentes nas quais as TIC representam um elemento transversal. Vários estudos abordaram a inteligência das cidades, mas poucos com ênfase em governança das TIC. Assim, este estudo analisou as práticas de governança de TIC em quinze cidades brasileiras mais bem classificadas no indice Cidades Inteligentes Conectadas. A metodologia abrangeu pesquisa nos sites oficiais das cidades, nos portais de transparência e requisições de informação pela Lei Brasileira de Acesso à Informação. Os resultados evidenciaram que a maioria das prefeituras carece de uma abordagem de programa para cidades inteligentes e de boas práticas de governança de TIC, bem como algumas cidades não evidenciaram conformidade com a Lei de Acesso à Informação.

\section{Introdução}

A Transformação Digital (TD) surgiu como um fenômeno importante de pesquisa na área de Sistemas de Informação [Bharadwaj et al. 2013] e para profissionais [Fitzgerald et al., 2014]. Em alto nível, a TD abrange as mudanças que ocorrem na sociedade com o uso de tecnologias digitais [Majchrzak et al. 2016]. Em nível 
organizacional, a TD tem argumentado que as organizações devem identificar formas de inovar com as novas tecnologias, elaborando estratégias para alcançar um melhor desempenho operacional [Hess et al. 2016].

Reconhecendo as oportunidades da TD no Brasil, o governo publicou a Estratégia Brasileira de Transformação Digital [MCTIC 2018]. Considerando que o país é composto por um distrito federal, 26 estados e 5.570 municípios [IBGE 2017], esta publicação fornece um conjunto de ações para ampliar a transparência e o controle social visando a oferta de serviços públicos digitais com qualidade [MCTIC 2018]. Nesse cenário, os governos devem ter a capacidade de responder à TD com a ampliação de serviços inclusivos, convenientes e colaborativos para melhorar a confiança dos cidadãos [OCDE 2020]. Todavia, os municípios possuem autonomia política, administrativa e financeira incorrendo em desafios de governança multinível para coordenação de políticas digitais [OCDE 2018].

Segundo [Cunha et al. 2016], o governo pode melhorar a transparência com uso de TICs ampliando a publicidade de informações, automatizando processos e usando canais de comunicação entre gestores públicos e a sociedade. Todavia, esse movimento não ocorre de maneira homogênea nas cidades, pois existem diferentes níveis de maturidade de gestão e de governança de TIC. Assim, deve haver uma infraestrutura mínima de TIC e estrutura organizacional para gerenciar com eficácia projetos complexos [Ricart \& Ubaldi 2016] para superar a fragmentação de TIC [Laia et al. 2011]. Nesse sentido, a governança de TIC definida como uma estrutura para auxiliar as organizações públicas na decisão de TIC para alcançar o alinhamento entre TI e as partes interessadas em uma rede complexa representa um desafio para as cidades [Juiz \& Toomey 2015].

O conceito de cidade inteligente implica em uma gestão holística, visionária, colaborativa e transparente de recursos consubstanciada pela parceria público-privada, sistemas digitais, disseminação de informação aberta, priorização do capital humano, plataformas integradas, participação e colaboração dos cidadãos [Nam e Pardo 2011] [Gil-Garcia et al. 2015] [Rodríguez-Bolívar 2016]. Assim, as pesquisas científicas devem desenvolver modelos de governança para enfrentar os desafios da sociedade digital em relação à governança colaborativa, compartilhamento de informações, tomada de decisão baseada em evidências, envolvimento dos cidadãos, transparência e governo aberto [Pereira et al. 2018].

Todavia, poucos estudos enfocaram a governança de TIC em cidades inteligentes [Connolly et al. 2017] e não houve um esforço para entender como a governança de TIC é projetada, planejada, implementada e monitorada para alcançar o desempenho e a capacidade de resposta das cidades [Pereira et al. 2018]. Assim, há uma lacuna de pesquisa no estabelecimento de padrões e estruturas regulatórias [Ojo et al. 2015] e a necessidade de desenvolver pesquisas usando as TICs como um meta-fator [Chourabi et al. 2012][Gil-Garcia et al. 2015]. Conforme [Reis et al. 2021], os estudos atuais não fornecem uma base teórica sobre como as cidades inteligentes estão organizadas na perspectiva de governança de TIC.

Diante disso, este estudo teve como objetivo investigar o compromisso dos municípios brasileiros com práticas de governança das TIC como um fator para 
caracterizar a inteligência das cidades no contexto da transformação digital em quinze cidades melhor classificadas no Índice Connected Smart City 2020.

Este trabalho está organizado em seis seções, incluindo esta introdução. A seção 2 apresenta a revisão da literatura. A seção 3 apresenta a metodologia de pesquisa. A seção 4 consolida os dados. A seção 5 discute os resultados. A seção 6 apresenta as conclusões, limitações e trabalhos futuros.

\section{Revisão da Literatura}

\subsection{Governança de TIC}

A governança de TIC se refere a decisões de tecnologia e como elas são orquestradas para auxiliar os stakeholders e apoiar as estratégias das organizações [Huang et al. 2010]. Os mecanismos de governança de TIC devem ser aprovados e apoiados pela alta administração e cobrir toda a organização [Bianchi et al. 2017]. A composição da governança de TIC é baseada em estruturas (funções e responsabilidades) e processos (boas práticas), como COBIT e ITIL [Nfuka et al. 2010], e participação ativa e colaboração das partes interessadas [De Haes \& Grembergen 2009]. Esses componentes devem interagir para fornecer benefícios de governança eficazes para as organizações [Héroux \& Fortin 2017]. A governança de TIC representa uma questão crítica para evitar perdas estratégicas, financeiras e operacionais [Weill \& Ross 2004].

A governança de TIC também envolve processos de estruturação, práticas de trabalho e ambiente cultural [Weill \& Broadbent 2000]. Segundo o [SISP 2017], a governança das TIC é um sistema em que o uso atual e futuro das TIC é avaliado, dirigido e controlado para apoiar e monitorar os planos estratégicos e operacionais. A governança corporativa das TIC, exercida pelo conselho, deve ser baseada no alinhamento estratégico e na geração de valor [ISACA 2018].

Várias diretrizes e boas práticas sobre como a governança das TIC são implementadas surgiram da academia [De Haes \& Grembergen 2009] [Joshi et al. 2018]. Por exemplo, o COBIT 5 e o COBIT 2019 podem ser usados para estruturar a governança corporativa [ISACA 2018] [De Haes et al. 2019]. Em relação ao COBIT, [Reis et al. 2020] [Reis et al. 2020b] introduziram esta estrutura no contexto das cidades inteligentes, propondo um roteiro para implementar o framework neste cenário. No âmbito municipal, a governança de TIC está associada ao alinhamento entre a estratégia de TIC e seu Plano Diretor para garantir a melhoria contínua do serviço público para atendimento aos objetivos do plano orçamentário das cidades (LOA) [PwC 2015] [ISACA 2018] [Reis et al. 2020 e 2020b].

\subsection{Cidades Inteligentes}

Cidades inteligentes tem sido objeto de discussões na comunidade científica há algum tempo, mas pode ser considerado um tópico abstrato que carece de precisão [Hollands 2008] [Kobayashi et al. 2017]. Alguns autores definiram domínios para caracterizar as cidades como inteligentes propondo uma abordagem mais ampla para esse tópico, a exemplo de cidades inteligentes e sustentáveis que usam recursos de TIC [Ahvenniemi et al. 2017]. Outros abordaram as diferenças entre cidades inteligentes e cidades digitais, assumindo que um sistema inteligente está associado a um sistema digital [Jucevicius et al. 2014]. Segundo a ISO/IEC JTC 1, 
uma cidade inteligente aplica as novas tecnologias, como Internet das Coisas (IoT), computação em nuvem, big data e integração de informações geográficas para facilitar o planejamento, a construção, a gestão e a entrega de serviços inteligentes aos cidadãos [ISO 2015].

Alguns autores associaram o conceito de cidades inteligentes a modelos ou frameworks [Nam e Pardo 2011]. [Gil-Garcia et al. 2015] descreveu as principais características das cidades inteligentes em uma estrutura composta por quatro dimensões e dez domínios: Dados e Tecnologia (Dados e Informação; TIC e outras Tecnologias); Ambiente Físico (Ambiente Construído e Infraestrutura da Cidade; Ambiente Natural e Sustentabilidade Ecológica); Sociedade (Governança, Engajamento e Colaboração; Capital Humano e Criatividade; Economia do Conhecimento e Ambiente Pró-Negócios); Governo (Serviços Públicos; Administração e Gestão Municipal; Arranjos Institucionais).

No contexto brasileiro, o Plano Diretor de Tecnologia da Informação das Cidades Inteligentes (PDTCI) é um instrumento que descreve os desafios das cidades a partir da visão preconizada pela prefeitura para a implementação de soluções inteligentes de acordo com o planejamento de execução [BNDES 2018]. Diante disto, as TIC podem expandir o potencial econômico de uma região e criar competitividade, levando a oportunidades de inovação e possibilitando a transformação inteligente dos municípios [Przeybilovicz et al. 2018]. Diante disso, a Sociedade Brasileira de Computação enquadrou o tema cidades inteligentes como um desafio de pesquisa para a comunidade, sendo a governança de TIC um elemento fundamental [Boscarioli et al. 2017].

\section{Metodologia e Procedimentos de Pesquisa}

A metodologia de pesquisa baseou-se em um estudo aplicado e descritivo [Lakatos \& Marconi 2006], com uso de dados disponíveis como evidências [Benbasat et al. 1987] e estruturada em seis Fases conforme Figura 1.

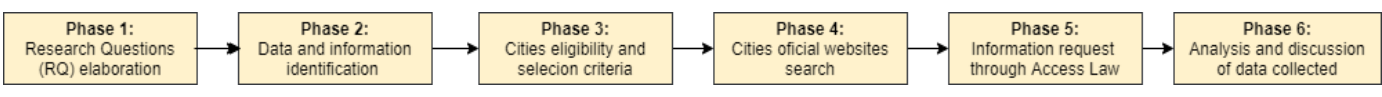

Figura 1: Fases da metodologia de pesquisa. Elaborado pelos autores

A Fase 1 compreendeu a formulação de duas Questões de Pesquisa (RQ): RQ\#1: Como os municípios brasileiros institucionalizam, gerenciam e monitoram seus programas de cidades inteligentes? e RQ\#2: Como as práticas de governança de TIC estão estruturadas nesses municípios? A Fase 2 compreendeu o levantamento de informações baseado em [Chourabi et al. 2012][ISO 2015b][MCTIC 2018][ISACA 2018][Reis et al. 2020 e 2020b] para a análise das práticas de governança de TIC. A Tabela 1 relaciona as informações selecionadas.

Tabela 1: Dados e informações para o estudo. Elaborado pelos autores

\begin{tabular}{|l|l|}
\hline ID & Descrição dos dados e informações \\
\hline$\# 1$ & Programa, projeto, plano ou documento semelhante de cidade inteligente contendo iniciativas inteligentes de cidades \\
\hline$\# 2$ & Planos diretores e estratégicos de TIC e outros instrumentos relacionados \\
\hline$\# 3$ & Modelo de governança de TIC identificando áreas, responsabilidades e processos \\
\hline$\# 4$ & Estrutura de governança de TIC \\
\hline$\# 5$ & Política de governança de TIC \\
\hline$\# 6$ & Comitês de TIC descrevendo seus nomes, objetivos, competências e composição \\
\hline$\# 7$ & Benefícios e indicadores de governança de TIC \\
\hline
\end{tabular}


A Fase 3 compreendeu a seleção da amostra das cidades que considerou os 15 municípios com mais de 500 mil habitantes melhor classificados no Ranking Connected Smart Cities 2020 [Smart Cities 2020] em 25/07/2020. Este ranking mapeia as cidades inteligentes com potencial de desenvolvimento por setenta indicadores. $\mathrm{Na}$ ordem do ranking, as cidades selecionadas foram: $1^{\circ}$ São Paulo (SP), $2^{\circ}$ Florianópolis (SC), $3^{\circ}$ Curitiba (PR), $4^{\circ}$ Campinas (SP), $5^{\circ}$ Brasília (DF), $6^{\circ}$ Porto Alegre (RS), $7^{\circ}$ Belo Horizonte (MG), $8^{\circ}$ Niterói (RJ), $9^{\circ}$ Rio de Janeiro (RJ), $10^{\circ}$ Campo Grande (MS), $11^{\circ}$ Recife (PE), $12^{\circ}$ São José dos Campos (SP), $13^{\circ}$ Joinville (SC), $14^{\circ}$ Londrina (PR), $15^{\circ}$ Salvador (BA) e $16^{\circ}$ São Bernardo do Campo (SP). A capital Brasília, na $5^{\mathrm{a}}$ posição, por ser considerada uma região administrativa do Distrito Federal, com características únicas de administração foi excluída da amostra e São Bernardo do Campo na $16^{a}$ posição foi incluída.

A Fase 4 compreendeu acesso aos sites das prefeituras e aos portais de transparência para consulta às informações da Tabela 1. A Fase 5 compreendeu a solicitação das informações aos municípios com base na Lei de Acesso à Informação entre 01/03/2020 e 30/11/2020 usando o e-SIC (Serviço de Informação Eletrônica ao Cidadão) [Brasil 2009] [Brasil 2011]. A Fase 6 compreendeu a consolidação, análise e discussão dos dados.

\section{Análise dos Dados}

Os dados consolidados estão descritos na Tabela 2.

Tabela 2: Consolidação dos dados. Elaborado pelos autores

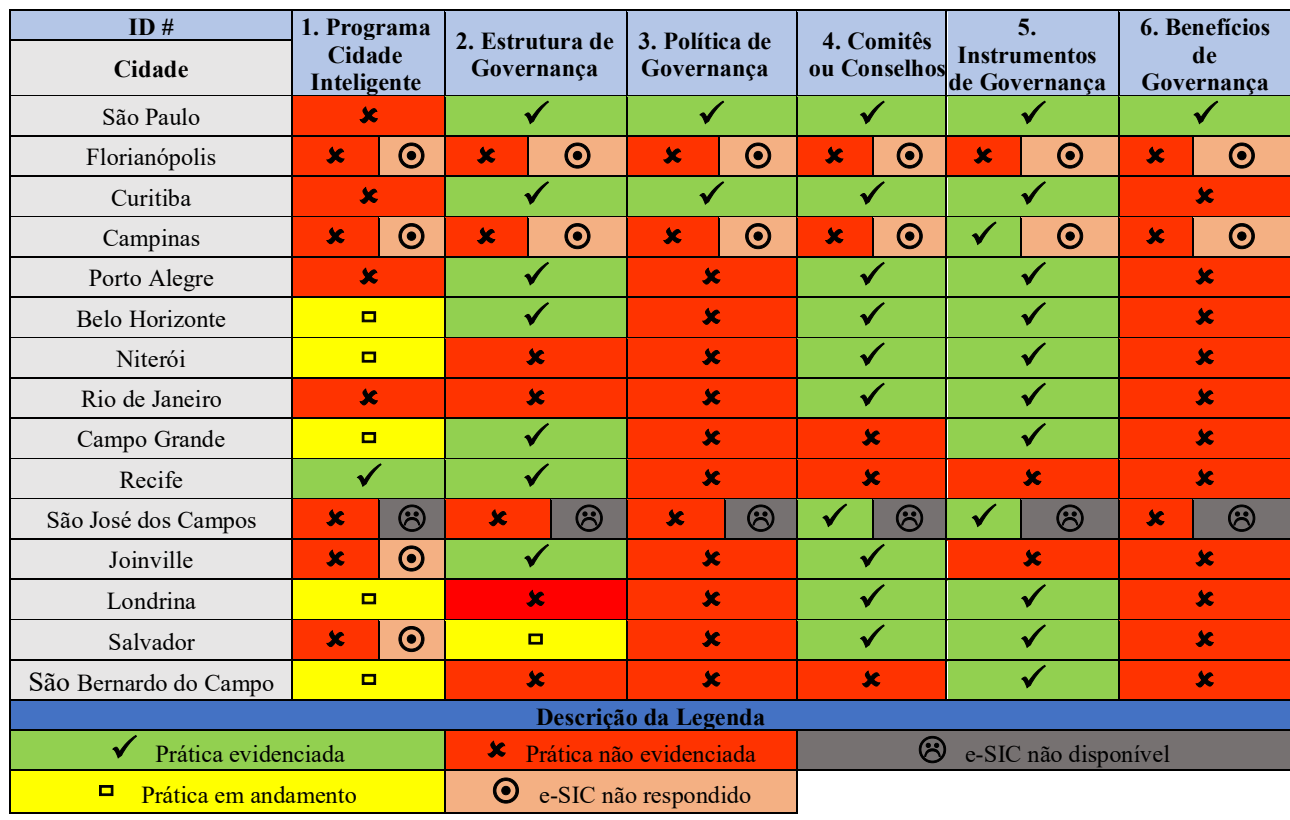

O símbolo " $\checkmark$ " em verde indica que a prática foi evidenciada. O símbolo “口" em amarelo indica que a prática está em andamento, mas não implementada. O símbolo " $x$ " em vermelho indica que a prática não foi evidenciada. O símbolo “๑” em laranja indica que o e-SIC não foi respondido. O símbolo ":: em cinza indica que a funcionalidade e-SIC não está disponível no site. 
Recife foi a única cidade que apresentou um programa de cidade inteligente que está sendo executado pela sociedade organizada "Centro de Gestão Digital do Porto e Cidadãos". O marco desse programa é 12/03/2037, data de comemoração de 500 anos da cidade. O programa é uma parceria entre a prefeitura e a Agência de Inovação e Estratégia de Recife (AIRES). O programa de cidades inteligentes de Belo Horizonte, constante do Plano Plurianual de Ação Governamental (PPAG), encontra-se em avaliação para submissão como projeto de lei. O programa de cidade inteligente de Niterói está sendo planejando em parceria com a Universidade Federal Fluminense (UFF), em alinhamento ao Programa de Desenvolvimento de Projetos Aplicados (PDPA) da cidade. Campo Grande instituiu um grupo de trabalho para definir as diretrizes para o seu programa de cidade inteligente constate do Plano Diretor de Desenvolvimento Urbano Ambiental (PDDUA). Londrina contratou uma consultoria especializada para elaborar seu Plano Estratégico (Plano Diretor) e uma minuta de Decreto para estabelecer os direcionadores que nortearão seu Programa Cidade Inteligente, sendo 2040 o marco. São Bernardo do Campo está trabalhando em vários projetos para consolidar um programa de cidade inteligente que contemplam iniciativas para atendimento aos cidadãos, sistema de monitoramento inteligente e segurança. São Paulo informou que uma visão de uma cidade inteligente e humana foi construída em 2019, mas o atual governo não deu continuidade ao projeto. Curitiba tem várias iniciativas em seu Plano Diretor de TIC voltadas para cidades inteligentes, mas sem uma visão de programa. O Rio de Janeiro também relatou diversas iniciativas inteligentes em vigor: Cidade do Aprendizado, Cidade Atual, Central 1746, Plataforma do Cidadão Colaborativo e Centro de Operações do Rio (COR). São José dos Campos não possui recurso online (e-SIC) para solicitar informações, mas no site havia um projeto inteligente San José, composto pelo Novo Centro de Segurança e Inteligência, Câmeras Integradas de Inteligência, Wi-Fi Gratuito e Salas de aula conectadas. Campinas não respondeu ao e-SIC, mas seu Plano Estratégico Cidade Inteligente (PECCI) 2019-2029 no website, descreve as iniciativas da cidade. Florianópolis, Joinville e Salvador não responderam ao e-SIC e as informações não estavam disponíveis nos websites e portais de transparência.

Dentre as quinze cidades, oito não evidenciaram estruturas de governança de TIC em seus processos organizacionais. Em São Paulo, há uma Unidade Central que coordena a governança de TIC em ações descentralizadas. Em Curitiba, a Secretaria de Informação e Tecnologia (SIT) coordena e orienta as ações descentralizadas por meio dos Núcleos Setoriais de Informação e Tecnologia (NITs). Em Porto Alegre, a Secretaria Municipal de Planejamento e Gestão (SMPG) é responsável pela governança de TIC e Geoprocessamento. Em Belo Horizonte, a Câmara de Coordenação Geral (CCG), é o órgão central de governança, incluindo as administrações direta e indireta. Em Campo Grande, a governança e a gestão das TIC são centralizadas na Agência Municipal de Tecnologia da Informação e Inovação (AGETEC), unidade da administração indireta. Em Recife, a Empresa Municipal de Informática (EMPREL), empresa pública da administração indireta, é responsável pela governança das TIC na cidade. A governança de TIC na cidade de Joinville é de responsabilidade de uma Unidade de Gestão, vinculada à secretaria de administração e planejamento. As demais cidades não possuem uma estrutura de governança de TIC. 
Em relação às políticas de governança de TIC, São Paulo e Curitiba foram as únicas cidades que evidenciaram esses artefatos. A política de São Paulo visa proporcionar melhoria da maturidade; planejamento, execução, monitoramento e padrões técnicos; uso estratégico de tecnologias; gerenciamento e integração de banco de dados; processos inovadores baseados nos cidadãos; e uso racional de recursos com base na responsabilidade social. A política de Curitiba é composta pelo uso de TIC, aquisição de soluções de TIC, planos setoriais de TI (PSTI), soluções e regulamentos de serviços de TIC, arquitetura tecnológica corporativa, gestão de serviços de TIC, desenvolvimento e suporte de sistemas. Salvador emitiu uma Política Municipal de TIC, mas essa política somete será publicada após a sua aprovação pela Câmara Municipal. As demais cidades não evidenciaram políticas de governança de TIC.

Em relação aos comitês elou conselhos de governança de TIC, Florianópolis, Campinas, Recife, Campo Grande, Joinville e São Bernardo do Campo não evidenciaram sua existência. Campinas e Joinville instituíram conselhos municipais, mas não há responsabilidades atribuídas à governança das TIC. Recife instituiu três comitês, mas nenhum está relacionado à governança de TIC. As demais cidades evidenciaram os comitês e/ou conselhos de governança.

Em relação aos instrumentos de governança de TIC, Florianópolis, Joinville e São Bernardo do Campo não forneceram evidências sobre a adoção desses artefatos. São Paulo, Curitiba, Belo Horizonte, Rio de Janeiro e Niterói definiram instrumentos de governança de TIC estratégicos (Plano Estratégico de TIC, Plano de Investimentos e Plano de Inovação, por exemplo) e operacionais (Plano Setorial de TI, Plano de Gestão de Serviços e Guias Técnicos, por exemplo), enquanto em Porto Alegre os instrumentos eram estritamente operacionais. Recife e Campo Grande atribuíram a responsabilidade de governança a empresas de sua Administração Indireta. Campinas foi a única cidade que evidenciou o Plano Estratégico de Cidade Inteligente como um artefato para alinhar cidade inteligente e governança de TIC definindo diretrizes para a transformação da cidade.

Em relação aos benefícios da governança das TIC, São Paulo foi a única cidade que relatou economia com menor custo, mantendo a qualidade e agilidade no atendimento da gestão de bens públicos; aumento da maturidade dos processos; e cumprimento das metas do Plano Estratégico. Esse modelo reforça a autonomia das secretarias, submunicípios e da administração indireta em relação às TI, sem diminuir a relevância estratégica de empresa municipal PRODAM.

\section{Discussão dos Resultados}

A visão das cidades em programa de cidade inteligente foi limitada a iniciativas isoladas de cada secretaria municipal na forma de "silos". A maioria dos municípios informou sobre a importância de um ambiente inteligente e sustentável para as cidades do futuro, embora um programa de cidade inteligente esteja em vigor apenas em Recife. Um programa de cidade inteligente atua como uma estrutura holística para coordenar iniciativas focadas nos desafios das cidades para estimular sua infraestrutura tecnológica, construir uma base para uma gestão integrada, aumentar o desenvolvimento econômico e a geração de soluções integradas com uso de novas tecnologias alinhadas às necessidades dos cidadãos. 
As estruturas de governança de TIC das cidades são distintas umas das outras. A maioria das cidades está adotando ações e decisões descentralizadas, coordenadas por um órgão central, embora Rio de Janeiro e Niterói relataram dificuldades nesse processo. São Paulo foi a única cidade que evidenciou uma abordagem holística de governança de TIC com base em atividades monitoradas para avaliar a eficácia dos serviços. Belo Horizonte reforçou que seus entes municipais estão proibidos de executar iniciativas tecnológicas sem autorização da Câmara. Por outro lado, Campo Grande e Recife atribuíram responsabilidades de governança às empresas da Administração Indireta. Florianópolis, Campinas, Niterói, Rio de Janeiro, São José dos Campos, Salvador e São Bernardo do Campo não evidenciaram estruturas de governança de TIC.

Uma política de governança de TIC representa um conjunto de objetivos e diretrizes para alinhar ações estratégicas no uso de recursos de TIC nas cidades. Nas cidades inteligentes, as políticas devem promover a cidadania digital por meio de ações governamentais de transparência, aumento dos serviços eletrônicos, por um processo de gestão pública municipal eficaz. Esta prática precisa evoluir no contexto das cidades, pois foi evidenciada somente em São Paulo e Curitiba.

As funções dos comitês de governança de TIC da maioria das cidades são extensivas às administrações direta e indireta e composta por um grupo deliberativo com membros de diversas secretarias das cidades. O Rio de Janeiro institucionalizou quatro comitês de governança para discutir temas relacionados a TIC e inovação, enquanto Belo Horizonte estabeleceu um comitê para iniciativas específicas da secretaria de saúde.

Os instrumentos de governança de TIC representam artefatos usados para estruturar as diretrizes de governança e monitorar o cumprimento de metas. Os frameworks COBIT 2019, ITIL e SISP fornecem um conjunto de artefatos de governança que podem ser usados pelas organizações ao projetar seus modelos de governança. Algumas cidades adotaram instrumentos de governança estratégica e operacional e outras foram documentos estritamente operacionais. Campinas foi a cidade única que evidenciou um Plano Estratégico de Cidade Inteligente para alinhar questões de governança de TIC e transformá-la em uma cidade inteligente, humana e sustentável. Embora alguns municípios tenham institucionalizado instrumentos de governança de TIC, quando comparados com boas práticas e padrões de governança pública, suas adoções estão em um estágio inicial e as cidades ainda não estão usando a maioria das estruturas, padrões e capacidades propostas por acadêmicos e profissionais no setor público.

São Paulo foi a única cidade que publicou dados de governança ativa em seu portal. A cidade utiliza painéis para monitorar a entrega de serviços e relatou um estudo aplicado à gestão do parque de microinformática sobre benefícios da governança de TIC. Nesse caso, a empresa municipal de tecnologia PRODAM vinha apoiando as entidades na atualização de seus parques tecnológicos, disponibilizando registros de preços públicos. Todavia, essa abordagem evidenciou uma demanda de serviço em que a PRODAM não era a melhor opção, portanto passou a permitir que cada município negociasse contratos com fornecedores. Como resultado, houve uma melhoria do indicador de ativos de microinformática 30\% em 2017 para 68\% em 2020. 


\section{Conclusão e Trabalhos Futuros}

Este estudo analisou as práticas de governança de TIC em quinze cidades brasileiras com mais de 500 mil habitantes e melhores classificadas no ranking Connected Smart City. Primeiramente, foi identificado um conjunto de dados a serem avaliados, cuja metodologia englobou buscas nos sites e portais de transparência das cidades, bem como requisições via Lei Brasileira de Acesso à Informação. Este estudo demonstrou fragilidades na adoção de práticas de governança das TIC para a transformação inteligente das cidades.

Florianópolis e Campinas não cumpriam a Lei Brasileira de Acesso à Informação por não atenderem à requisição via e-SIC. São José dos Campos não disponibilizou a função e-SIC em seu portal. São Paulo foi a única cidade que evidenciou transparência ativa em relação à governança das TIC e uma minoria de informações solicitadas estava disponível proativamente nos sites ou portais de transparência das demais cidades. O conceito de cidades inteligentes brasileiras está limitado a iniciativas de cada secretaria, em visões isoladas, não integradas em um programa holístico e coerente, tornando um desafio de gestão a coordenação dos benefícios advindos. Campo Grande, Belo Horizonte e Niterói evidenciaram a intenção de estruturar um programa de cidade inteligente alinhado ao Plano Diretor das cidades. O desenvolvimento do Plano Estratégico de Cidade Inteligente de TIC em conjunto com academia, cidadãos e indústrias, conforme evidenciado em Campinas, funciona como uma boa prática que as cidades devem adotar no contexto de um programa de cidade inteligente. Assim, as cidades brasileiras devem buscar uma visão de um programa de gestão inteligente por um conjunto de projetos coordenados com objetivos comuns.

A estrutura de governança de TIC de algumas cidades, compreendendo a composição de comitês, políticas e instrumentos de governança para o ambiente de transformação digital não estavam em alinhamento com as práticas de governança adotadas como referência neste estudo. Por exemplo, as políticas de TIC não foram concebidas na maioria das cidades; Comitê e estrutura de governança de Recife e Campo Grande foram atribuídos à Administração Indireta Municipal; $\mathrm{O}$ conselho de Campinas, exclusivamente consultivo, não está em operação para atender às necessidades locais; O comitê de TIC de Belo Horizonte foi concebido exclusivamente para iniciativas da saúde. Os comitês representam as instâncias municipais com o objetivo de avaliar as necessidades e o uso de tecnologia para as cidades, sob a perspectiva estratégica de novos serviços. Assim, um comitê deve acompanhar o progresso da tecnologia, priorizar projetos, identificar necessidades de orçamento, aprovar políticas e questões de segurança, incentivando a participação dos cidadãos em uma abordagem de governança aberta. Verificou-se que as funções de TI das cidades variam de acordo com sua estrutura. No entanto, com base na descentralização de TIC nas secretarias, os mecanismos de governança corporativa de TIC devem ser implementados nesse processo. Diante disso, este trabalho evidenciou vulnerabilidades na concepção da governança das cidades, de modo que os gestores públicos e profissionais podem utilizar este estudo para planejar práticas de governança locais.

Uma limitação deste estudo foi que as práticas de governança foram analisadas, à luz de sua concepção, sob os aspectos de compliance que abrangem a 
governança das TIC em frameworks. Trabalhos futuros podem aplicar estudos de caso para avaliar a eficácia da governança nas cidades, desenvolver um processo para apoiar a governança aberta de TIC utilizando meta dados e desenvolver um catálogo de práticas de governança de TIC. Uma análise mais aprofundada pode incluir pesquisas com especialistas e entrevistas com membros do governo para compreender os desafios da adoção de práticas de governança de TIC nas cidades.

Este trabalho contribui com a comunidade científica e profissionais da área de governo digital das cidades brasileiras e pode auxiliar gestores públicos a aplicar práticas de governança de TIC em grandes e médias cidades. A governança de TIC consiste em liderança, políticas, padrões, estrutura organizacional, métodos e processos para garantir que a TI apoie e aprimore os objetivos e estratégias das cidades, agregando valor aos serviços públicos sob responsabilidade da Alta Administração. Portanto, a governança nas cidades inteligentes deve ser liderada pelo prefeito e orientada pelo corpo executivo das cidades por meio de programas alinhados às necessidades dos cidadãos.

\section{Referências}

Ahvenniemi, H., Huovila, A., Pinto-Seppä, I., \& Airaksinen, M. (2017). What are the differences between sustainable and smart cities? Cities, 60, 234-245.

Benbasat, I., Goldstein, D. K., \& Mead, M. (1987). The Case Research Strategy in Studies of Information Systems. MIS Quarterly, 11(3), 369.

Bianchi, I. S., Sousa, R. D., Pereira, R., Luciano, E. (2017). IT Governance Structures in Brazilian, Dutch and Portuguese Universities. Procedia Computer Science, 121, 927-933. https://doi.org/10.1016/j.procs.2017.11.120.

BNDES (2018). Estudo: Internet das Coisas: um Plano de Ação para o Brasil.

Boscarioli, C.; Araujo, R. M.; Maciel, R. S. P. (2017). I GranDSI-BR - Grand Research Challenges in Information Systems in Brazil 2016-2026. Special Committee on Information Systems (CE-SI). Brazilian Computer Society.

Brasil (2009). Lei Complementar 131/2009. Transparência e Responsabilidade Fiscal. Disponível em https://abre.ai/cwG4.

Brasil (2011). Lei $\mathrm{n}^{\circ} 12.527 / 2011$. Acesso a informações. Disponível em http://www.planalto.gov.br/ccivil_03/_ato2011-2014/2011/lei/112527.htm.

Bharadwaj, A., El Sawy, O., Pavlou, P., Venkatraman, N. (2013). Digital business strategy: Toward a next generation of insights. MIS Quarterly (37:2), pp. 471482.

Chourabi, H., Nam, T., Walker, S., Gil-Garcia, J. R., Mellouli, S., Nahon, K., Scholl, H. J. (2012). Understanding Smart Cities: An Integrative Framework. 2012 45th Hawaii International Conference on System Sciences.

Connected Smart Cities. (2020). Urban Systems. Available at https://abre.ai/cwG6

Connolly, N., G. Maccani and B. Donnellan. (2017). IT Governance in Smart Cities: A Conceptual Framework. ICIS.

Cunha, M. A., Coelho, T. R., Silva, T. A. B., Cantoni, S. L., \& Teixeira, M. A. C. (2016). Government transparency in the Brazilian Federation: Heterogeneous results originating from different IT capacities. In Brazilian Internet Steering Committee - CGI.br. Survey on the use of information and communication technologies in the Brazilian public sector: ICT Electronic Government 2015. 
De Haes, S. \& Grembergen, W. V. (2009). An Exploratory Study into IT Governance Implementations and its Impact on Business/IT Alignment, Information Systems Management, 26:2, 123-137.

De Haes S., Van Grembergen W., Joshi A., Huygh T. (2019) COBIT as a Framework for Enterprise Governance of IT. In: Enterprise Governance of Information Technology. Management for Professionals. Springer, Cham.

Fitzgerald, M., Kruschwitz, N., Bonnet, D., and Welch, M. (2014). Embracing digital technology: A new strategic imperative. MIT Sloan Management Review (55:2), pp. 1-12.

Gil-Garcia, J. R.; Pardo, T. A.; Nam, T. (2015). What makes a city smart? Identifying core components and proposing an integrative and comprehensive conceptualization. Information Polity, v. 20, n. 1, p. 61- 87, 2015.

Héroux, S.; Fortin, A. (2017). Exploring the influence of executive management diversity on IT governance. CHAIRE DínformationFinanciére et Organisationnelle ESQ UQÀM, (514), 1-33.

Hess, T., Matt, C., Benlian, A., and Wiesboeck, F. (2016). Options for formulating a digital transformation strategy. MIS Quarterly Executive (15:2), pp. 123-139.

Hollands, R. G. (2008). Will the real smart city please stand up? Intelligent, progressive or entrepreneurial? City, 12(3), 303-320.

Huang, R., Zmud, R.W., Price, R.L. (2010). Influencing the effectiveness of IT governance practices through steering committees and communication policies, European Journal of Information Systems, 19(3), pp.288-302.

IBGE (2017) Instituto Brasileiro de Geografia e Estatística, IBGE. Disponível em https://cidades.ibge.gov.br/brasil/panorama.

ISACA (2018). COBIT 2019 Framework: Introduction and Methodology.

ISO/IEC JTC 1 (2015). International Electrotechnical Commission (IEC). Smart Cities, Preliminary Report.

ISO/IEC 38500 (2015b). Information technology - governance of IT.

Joshi, A., Huygh, T., Haes, S., Grembergen, W. (2018). An Empirical Assessment of Shared Understanding in IT Governance Implementation. HICSS.

Jucevicius, R., Patašiene, I., Patašius, M. (2014). Digital Dimension of Smart City: Critical Analysis. Procedia - Social and Behavioral Sciences, 156.

Juiz, C., \& Toomey, M. (2015). To govern IT, or not to govern IT? Communications of the ACM, 58(2), 58-64.

Kobayashi, A. R. K., Kniess, C. T., Serra, F. A. R., Ferraz, R. R. N., Ruiz, M. S. (2017). Cidades inteligentes e sustentáveis: estudo bibliométrico e de informações patentárias. International Journal of Innovation, 5(1), 77-96.

Laia, M. M., Cunha, M. A. V. C., Nogueira, A. R. R., \& Mazzon, J.A. (2011). Electronic government policies in Brazil: Context, ICT management and outcomes. Revista de Administração de Empresas, 51(1), 43-57.

Lakatos, E. M.; Marconi, M.A. (2006). Fundamentos de Metodologia Científica. In: 6 São Paulo: Ed. Atlas.

Majchrzak, A., Markus, M. L., and Wareham, J. (2016). Designing for digital transformation: Lessons for information systems research from the study of ICT and societal challenges. MIS Quarterly (40:2), pp. 267-277.

MCTIC. Ministério da Ciência, Tecnologia, Inovações e Comunicações. (2018). Brazilian Digital Transformation Strategy: E-Digital, Brasília. 
Nam, T.; Pardo T. (2011). Conceptualizing Smart City with Dimensions of Technology, People, and Institutions, In Proceedings of the 12th Annual International Digital Government Research Conference.

Nfuka, Edephonce N. and L. Rusu. (2010). Critical success factors for effective IT governance in the public sector organizations in a developing country: The case of Tanzania. In Proceedings of the 18th European Conference on Information Systems (ECIS), Pretoria, South Africa, June 7-9.

OECD (2018). Digital Government Review of Brazil: Towards the Digital Transformation of the Public Sector, OECD Digital Government Studies.

OECD (2020). Going Digital in Brazil, OECD Reviews of Digital Transformation, OECD Publishing, Paris, https://doi.org/10.1787/e9bf7f8a-en.

Ojo, A., Dzhusupova, Z., \& Curry, E. (2015). Exploring the Nature of the Smart Cities Research Landscape. In R. Gil-Garcia, T. A. Pardo, \& T. Nam (Eds.), Smarter as the New Urban Agenda. Springer.

Pereira, G. V., Eibl, G., Parycek, P. (2018). The Role of Digital Technologies in Promoting Smart City Governance. In Companion Proceedings of the Web Conference 2018 (WWW '18). International World Wide Web Conferences.

Przeybilovicz, E., Cunha, M. A., \& Meirelles, F. S. (2018). The use of information and communication technology to characterize municipalities: who they are and what they need to develop e-government and smart city initiatives. Revista de Administração Pública, 52(4), 630-649.

PwC (2015) PricewaterhouseCoopers. King III IT Governance in Organization.

Reis, L. C. D.; Ferreira, S. B. L., Bernardini, F. C., Cappelli, C. (2020) Towards a COBIT5 approach to ICT governance requirements in smart cities. In Proceedings of the $13^{\text {th }}$ International Conference on Theory and Practice of Electronic Governance (ICEGOV 2020).

Reis, L. C. D, Bernardini, F. C, Cappelli, C, Ferreira, S. B. L. (2020b) ICT Governance in the Context of Smart Cities: An Exploratory Study in Municipalities in the state of Rio de Janeiro. In: Proceeding $8^{\text {th }}$ WCGE 20 SBC

Reis, L. C. D., Bernardini, F. C, Cappelli, C, Ferreira, S. B. L. (2021). An ICT governance analysis for the digital and smart transformation of Brazilian municipalities. In DG.O2021. DOI:https://doi.org/10.1145/3463677.3463729

Ricart, R. M., \& Ubaldi, B.C. (2016). Designing digital government to support inclusive and sustainable growth in Latin America and the Caribbean. In Brazilian Internet Steering Committee - CGI.br. Survey on the use of information and communication technologies in the Brazilian public sector.

Rodríguez-Bolívar, M. P. (2016). Characterizing the Role of Governments in Smart Cities: A Literature Review. In: Gil-Garcia J., Pardo T., Nam T. (eds) Smarter as the New Urban Agenda. Public Administration and Information Technology, vol 11. Springer, Cham.

SISP. Guia de Governança de TIC. (2017). Ministério do Planejamento, Desenvolvimento e Gestão. Disponível em http://www.sisp.gov.br/.

Weill, P. and Ross, J. W. (2004). IT Governance on One Page. MIT Sloan Working Paper No. 4517-04; CIS Research Working Paper No. 349.

Weill, P., Broadbent, M. (2000). Managing IT infrastructure: a strategic choice. In: Zmud, R., (Ed.), Framing the Domains of IT Management, Cincinnati, OH. 